\section{Serum Albumin: A Promising Biomarker to Anticipate Postoperative Complications}

To the Editor-We have read the article of $\mathrm{Ge}$ et al ${ }^{1}$ with particular interest. This study tested the predictive value of postoperative decrease of serum albumin (SA) for adverse outcomes in a retrospective cohort of colorectal surgery. Of note, the concept suggesting that $\Delta$ albumin is a predictor of complications is not exactly novel. ${ }^{2-4}$ It is, however, critical to validate the role of $\Delta$ albumin, particularly because it is an easy and inexpensive biomarker, unlike other more complex ones. ${ }^{5,6}$ The authors established that a $15 \%$ reduction in SA within 2 postoperative days was an independent predictor of complications.

We address several points. First, did the authors test the predictive value of $\Delta$ albumin at postoperative day 1 because available data suggested that $\Delta$ albumin was already indicative at postoperative day $1 ?^{2,4}$ Second, did the authors compare the performance of absolute vs relative $\Delta$ albumin? In other words, does a reduction of $15 \%$ display a significantly better performance than, for example, $6 \mathrm{~g} / \mathrm{L}$ ? If not, one may assume that an absolute value may be easier to implement in clinical practice.

The authors also concluded that whether the conclusion of the current study could be extrapolated to other abdominal procedures, such as gastrectomy or esophagectomy resections, is unknown. Importantly, our group recently published a prospective study including all types of major abdominal surgery that confirmed that an early postoperative decrease of SA correlated with complications and length of stay. ${ }^{7}$ Strikingly, the protocol of our prospective study registered in clinicaltrials.gov (NCT02356484) in February 2015 shows surprising similarities with the retrospective study published in Diseases of the Colon \& Rectum Ge et al. ${ }^{1}$ Is this only by chance?

\section{REFERENCES}

1. Ge X, Dai X, Ding C, et al. Early postoperative decrease of serum albumin predicts surgical outcome in patients undergoing colorectal resection. Dis Colon Rectum. 2017;60:326-334.

2. Hübner M, Mantziari S, Demartines N, Pralong F, Coti-Bertrand P, Schäfer M. Postoperative albumin drop is a marker for surgical stress and a predictor for clinical outcome: a pilot study. Gastroenterol Res Pract. 2016;2016:8743187.
3. Ryan AM, Hearty A, Prichard RS, Cunningham A, Rowley SP, Reynolds JV. Association of hypoalbuminemia on the first postoperative day and complications following esophagectomy. J Gastrointest Surg. 2007;11:1355-1360.

4. Labgaa I, Joliat GR, Demartines N, Hübner M. Serum albumin is an early predictor of complications after liver surgery. Dig Liver Dis. 2016;48:559-561.

5. Rettig TC, Verwijmeren L, Dijkstra IM, Boerma D, van de Garde EM, Noordzij PG. Postoperative interleukin-6 level and early detection of complications after elective major abdominal surgery. Ann Surg. 2016;263:1207-1212.

6. Labgaa I, Demartines N, Hubner M. Biomarkers capable to early predict postoperative complications: the grail. Ann Surg. In press.

7. Labgaa I, Joliat GR, Kefleyesus A, et al. Is postoperative decrease of serum albumin an early predictor of complications after major abdominal surgery? A prospective cohort study in a European Centre. BMJ Open. In press.

Ismail Labgaa, M.D.

Nicolas Demartines, M.D.

Martin Hübner, M.D.

Lausanne, Switzerland

\section{The Authors Reply}

To the Editor-We would like to thank Labgaa et al for their interest regarding our article ${ }^{1}$ and the opportunity to respond to their comments.

First, receiver operating characteristic curve analysis was used to examine the predictive accuracy of $\Delta$ albumin at postoperative day (POD) 1 . The cutoff value of $\Delta$ albumin at POD 1 was $13.8 \%$ with area under the curve (AUC) 0.753 and Youden index 0.410. The result indicated that $\Delta$ albumin with nadir albumin within POD 2 performed better than $\Delta$ albumin at POD 1 in our study.

Second, the performance of absolute vs relative $\Delta$ albumin was compared using receiver operating characteristic curve analysis. The cutoff value of absolute $\Delta$ albumin was $5.95 \mathrm{~g} / \mathrm{L}$ in our study with AUC 0.782 and Youden index 0.472 . When relative $\Delta$ albumin was used, AUC was 0.810 and Youden index was 0.510. The result indicated that relative $\Delta$ albumin performed better than absolute $\Delta$ albumin in predicting complications after colorectal resection. ${ }^{1}$ This might be explained by the individual variation of baseline albumin level before surgery.

Dis Colon Rectum 2017; 60: e616-e618 DOI: 10.1097/DCR.0000000000000871 (c) The ASCRS 2017 